\title{
Optimal Routing for Safe Construction and Demolition Waste Transportation: A CVaR Criterion and Big Data Analytics Approach
}

\author{
Ying QIU, Xinna ZHAO, Xiaohong ZHANG
}

\begin{abstract}
Rapid urbanisation worldwide, especially in developing countries and areas, has led to the generation of large amounts of construction and demolition waste (C\&DW). The resultant transportation demands pose severe threats to safe transportation and secure city operation. By considering the low-probability-high-consequence nature of C\&DW traffic accidents and the effectiveness of route optimisation in transportation risk control, a risk-averse project was implemented. Furthermore, an optimal routing model based on the conditional value at risk (CVaR) criterion is proposed. The model considered various risk-averse attitudes of decision-makers. For practicality and for strongly supporting policy-making, big data technology, including the construction of multistructure databases and in-depth analysis, was applied to achieve the proposed CVaR routing model. Therefore, the present study extended the CVaR method to optimal routing design in the field of safe urban C\&DW transportation and integrated the optimal model with big data technology.
\end{abstract}

Keywords: big data technology; conditional value at risk (CVaR); construction and demolition waste (C\&DW); risk-averse attitude; routing

\section{INTRODUCTION}

The construction industry plays a large role in the establishment of emerging cities and metropolises. Large amounts of construction and demolition waste (C\&DW) are generated on the completion of massive building projects [1]. For example, in China, the amount of C\&DW is increasing by 1.8 billion tons per year, which has caused challenges to environmental protection, sustainable resource utilisation, and sustainable urban development [2]. The process of C\&DW removal has resulted in severe traffic accidents, which threaten the safe transportation and secure operation of cities and communities. Research indicates that routing design is an effective method to control potential transportation risks [3]. In practice, to avoid dangerous incidents, governments have begun to crack down on illegal C\&DW transportation or driver misconduct, especially through the supervision of construction trucks and prohibition of risky routing. However, ensuring safety in C\&DW transportation is difficult because the majority of C\&DW traffic accidents are caused by an irresponsible attitude and an insufficient understanding of the potential risks and consequences [4, 5].

We propose that two aspects should be further studied to form a research framework for C\&DW transportation management. For model construction, a decision-maker's risk-averse attitude and degree of impact considerably affect the optimal routing decision. Therefore, the conditional value at risk $(\mathrm{CVaR})$ method is introduced as a risk criterion to construct an optimal routing model for the field of C\&DW transportation. However, the application of big data technology provides excellent support for practical guidance through theoretical model simulation. A multistructure dataset including different sources of data enables easy and realistic model simulation and application.

\subsection{Routing Design and Decision-Maker's Risk Aversion}

Routing design is an effective method to control potential transportation risks [6]. Numerous achievements have been attained in routing model construction and precise and heuristic algorithm research [7, 8]. The classical vehicle routing problem (VRP) has been extended through the study of complex problems, such as vehicle routing with time windows $[9,10]$ and multicycle vehicle routing [11]. The study of VRP by considering risks has gradually emerged as a major research area [12]. The authors of [13] studied the multiyard vehicle path problem of explosive waste. They evaluated the environmental risks by building a dual-objective optimisation model. A multiobjective programming model was constructed considering transportation risks and costs [14] to study the route problem of heterogeneous vehicles for dangerous goods transportation. Moreover, a multiobjective genetic search algorithm was designed to solve the model. In [15], risky driver behaviours were examined. The authors of [15] determined through interviews and psychological tests that only self-discipline cannot be relied upon to prevent drivers from selecting high-risk paths. Safe urban transportation is treated as critical issue to ensure the development of sustainable cities and communities [16-18], which has been put forward as one of the sustainable development goals by UN. The risk factors in the aforementioned studies are all based on the assumption that the decision-maker is risk-neutral. Moreover, the objective impact of the decision-maker's risk aversion degree on the optimal path decision has not been considered in these studies.

The value at risk (VaR) theory has been widely used to model people's risk-averse attitude and risk-defending degree [19]. The authors of [20] established a road and transportation path optimisation model based on VaR to select an appropriate highway path for the transportation of dangerous goods. They used a heuristic algorithm to solve the model and analyse the influence of the decisionmaker's risk aversion on transportation path selection. In [21], the problem of selecting a transportation route for dangerous goods in railways was studied using the VaR risk model. Furthermore, the influence of a decisionmakers' risk aversion degree on the train configuration and train route selection was analysed. However, the tail risk is ignored in the VaR model, and accurate measurement of low-probability and high-hazard events beyond the VaR value is difficult. In addition, the VaR model does not have 
subadditivity and is not a consistent risk measurement criterion [22]. To overcome the aforementioned limitations, a complex CVaR model was introduced. Toumazis et al. [23] were the first to apply the CVaR criterion to road hazmat transportation. Hosseini and Verma [24] used the CVaR model in train configuration and route design for dangerous goods transportation. The effects of the $\mathrm{VaR}$ on the hazmat transportation were different from those on the train configuration [24].

However, limited research has been conducted on CVaR-based routing decisions. Most of the studies have focused on hazmat transportation [25, 26], which can barely regulate decision-maker behaviour or provide guidance in policy-making for safe C\&DW transportation.

\subsection{Routing Design and the Use of Big Data Technology}

Advancements in big data technology can equip cities and communities with transportation systems that can be managed using big-data-based optimal decision-making [27]. From the supervision perspective, big data technology can potentially improve the safety of transportation systems [28]. In addition, from the view of safe transportation guidance, big data such as traffic flow or traffic congestion notifications posted by governments or third-party platform can be regarded as alerts by drivers to make safe routing decisions [29]. A multiple-structure dataset is the most attractive feature of big data technology. This dataset is usually developed using real-time traffic information, historic urban planning, statistical population distribution, and crawled map data from open platforms. [30]. Research on the optimal route decision has resulted in two approaches. In the first approach, real-time guidance is provided to the drivers or autopilot system for directing the trajectory of vehicles [31]. In the second approach, the historical-data-based route planning or administrative provisions by enterprises and governments are considered. Compared with the first approach, the second approach is more comprehensive and more applicable for the transportation system as well as the integrated operation of the city. The majority of studies have mainly focused on human behaviour analysis or intelligent decision-making by using big data mining or forecasting methods [32]. However, studies should be conducted to examine how and to what extent the paradigms of big data technology can be extended to classical optimisation models in safe transportation.

To the best of our knowledge, this study is the first in which guidance and regulation of a decision-maker's routing decision is attempted. In this study, drivers' riskaverse attitudes were addressed using the CVaR routing model (CRM) and big data technology. The rest of this paper is organised as follows. In Section 2, we discuss the problem statement, including the complex C\&DW transportation system and the framework of model construction and big data absorption. In Section 3, the $\mathrm{CVaR}$ criterion and the proposed CRM is discussed in detail. In Section 4, an improved algorithm is proposed to transform the model. In Section 5, the case of urban C\&DW transportation routing design in Tongzhou District, Beijing, is studied using the proposed CRM, modified algorithm, and big data. Finally, in Section 6, the conclusion, managerial insights, and future research directions are outlined.

\section{PROBLEM STATEMENT}

Fig. 1 illustrates the overall recycling network of urban C\&DW, in which four stages are included and road transportation and large construction trucks play a crucial role. C\&DW recycling transportation networks inside cities are complex and difficult to regulate. For example, the Beijing Construction Committee has authorised 1240 sites for C\&DW landfill and recycling, 482 regulated enterprises, and 32880 heavy trucks to satisfy the legally controlled processing demand of C\&DW.

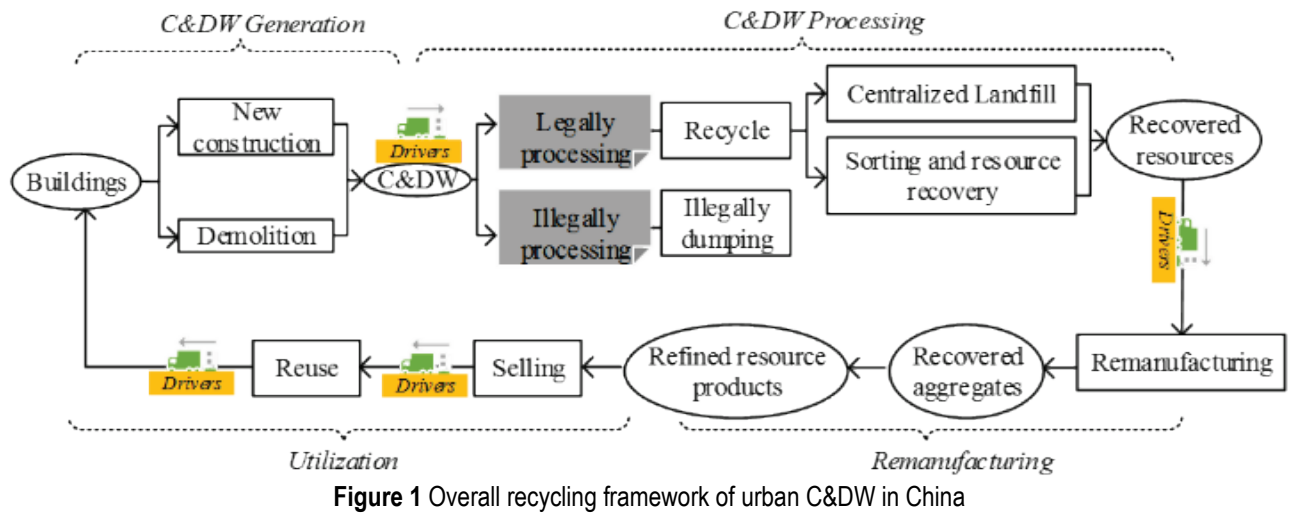

In the first section, we propose that the decisionmaker's (especially truck drivers) indifference to potential risks and ignorance of serious consequences are primary causes of accidents. The risk of accidents is aggravated in the complex transportation network. Population distribution is also considered as a critical factor in optimal routing decisions for traffic risk control. A trade-off should be devised between the avoidance of densely populated areas and the selection of the shortest path. Thus, densely populated areas should be consciously avoided to reduce and control the rates and consequences of accidents even if this requires the selection of longer routes and involves increased costs.

By using big data technology, companies and governments can better guide and regulate the behaviours of decision-makers. Therefore, we propose a multistructure dataset and a cross-research framework (Fig. 2) that combines the classical optimisation model with big data technology to extend theoretical studies in the field of safe C\&DW routing decisions. 


\section{MODELLING}

We assumed a directed and weighted transportation network $G(N, A)$, where $N$ represents the set of nodes and $A$ is the set of directed arcs. Consider a pair of origin and ending points $(O-D)$ in $G(N, A)$. According to the degree of risk aversion $(\alpha)$, decision-maker selects the optimal C\&DW transportation route with the minimum risk. The relevant mathematical notations and meanings are listed in Tab. 1.

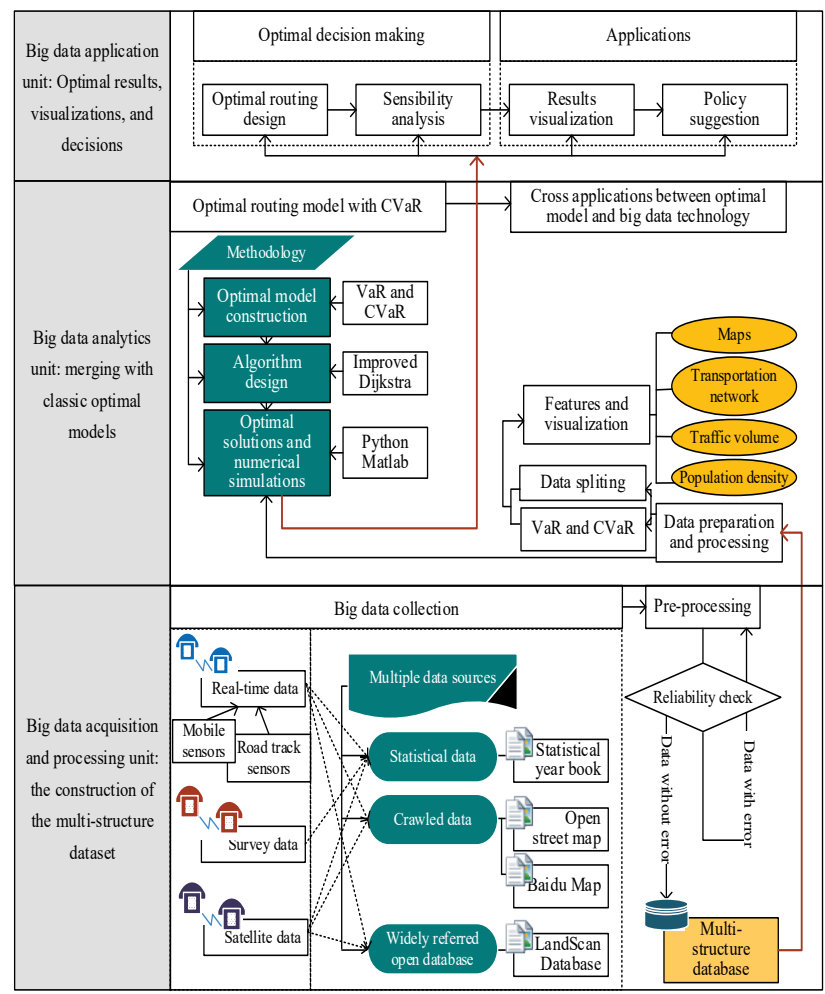

Figure 2 Multistructure dataset and framework devised in the study

Table 1 Mathematical notations and meanings

\begin{tabular}{|c|l|}
\hline Notations & \multicolumn{1}{c|}{ Meanings } \\
\hline$N$ & Set of nodes, indexed by $i, j(i, j \in N)$ \\
\hline$A$ & Set of directed arcs, indexed by $i, j(i, j) \in A$ \\
\hline$A_{i j}$ & Directed arc between node i and node $\mathrm{j}$ \\
\hline$L$ & Set of all alternative paths from $O$ to $D$ \\
\hline$l$ & One of the alternative paths, $l \in L$ \\
\hline$A^{l}$ & Set of all directed arcs of path $l$ \\
\hline$P_{i j}$ & $\begin{array}{l}\text { Probability of urban C\&DW transportation } \\
\text { incident on arc } A_{i j}\end{array}$ \\
\hline$C_{i j}$ & $\begin{array}{l}\text { Consequence from urban C\&DW transportation } \\
\text { incident on arc } A_{i j}\end{array}$ \\
\hline$\eta$ & $\begin{array}{l}\text { Critical value of consequence and cost for urban } \\
\text { C\&DW transportation accident, } \eta \in R^{+}, R^{+}=[0,+\infty)\end{array}$ \\
\hline$R^{l}$ & $\begin{array}{l}\text { Discrete random variable denoting the risk } \\
\text { associated with route } l\end{array}$ \\
\hline$x_{i j}$ & $\begin{array}{l}\text { Binary decision variable capturing routes of urban } \\
\text { C\&DW transportation }\end{array}$ \\
\hline$\alpha$ & $\begin{array}{l}\text { Specific confidence level, representing the } \\
\text { probability that the risk is not greater than a certain } \\
\text { value }\end{array}$ \\
\hline
\end{tabular}

Two types of factors should be considered in the construction of the CRM: (1) decision-makers' risk aversion and (2) tail risk avoidance and dangerous incident prevention. The construction of the CRM begins with the explication of the risk associated with one of the alternative paths $l$ selected from $G(N, A)$. For $l$, the cumulative distribution function of $R^{l}$ is critical [33]. The value of risk of $V a R_{\alpha}^{l}$ along path $l$ is defined with a specific confidence level $\alpha$ not exceeding a threshold $\eta$. According to the threshold, further assessment of $\mathrm{CVaR}_{\alpha}^{l}$ is achieved and the CRM is constructed.

\subsection{Risk Distribution and VaR Criterion}

We assumed that path $l$ contains a set of nodes $N^{l}$ and a set of directed arcs $A^{l}$ that guides the heavy trucks moving from $O$ to $D$. Here, $C_{(r)}^{l}$ denotes the $r^{\text {th }}$ smallest consequence value in the set $\left\{C_{i j}:\left(i, j \in A^{l}\right)\right\}$, and the corresponding accident probability is $P_{(r)}^{l}$ in the set $\left\{P_{i j}\right.$ : $(i$, $\left.\left.j) \in A^{l}\right\}\right\}$. Notably, $C_{(r)}^{l}$ is discretely distributed [20]. The corresponding discrete random variable $R^{l}$ can be defined according to (1) with the following values in ascending order, where $m \in 0,1,2, \ldots, r, \ldots, n_{l}: 0=C_{(0)}^{l}<C_{(1)}^{l}<C_{(2)}^{l}$

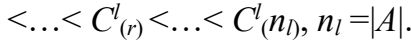

$$
R^{l}= \begin{cases} & 1-\sum_{m=1}^{n_{l}} P_{(m)}^{l} \\ C_{(0)}^{l} & \multicolumn{1}{c}{{ }^{l}} \\ C_{(1)}^{l} & P_{(1)}^{l} \\ C_{(2)}^{l} & P_{(2)}^{l} \\ \cdots & \ldots \\ C_{(r)}^{l} & P_{(r)}^{l} \\ \cdots & \ldots \\ C_{\left(n_{l}\right)}^{l} & P_{\left(n_{l}\right)}^{l}\end{cases}
$$

Probability of $R^{l}$ under a threshold $\eta$ is defined as $F_{R^{l}}(\eta)=\operatorname{Pr}\left(R_{l} \leq \eta\right)[20,21]$. The $\mathrm{VaR}$ along route $l$ for urban C\&DW transportation when considering decisionmakers' risk aversion degree is defined as follows:

$$
V a R_{\alpha}^{l}=\min \left\{\eta \mid F_{R^{l}}(\eta) \geq \alpha\right\}=\min \left\{\eta \mid \operatorname{Pr}\left(R^{l} \leq \eta\right) \geq \alpha\right\}
$$

\subsection{CVaR Criterion and the Proposed CRM}

For a path $l \in L$ in the network $G=(N, A)$, we established the CRM by considering the minimum value of $C_{V a R}^{l}$ at the specific confidence level $\alpha$ as $C V a R_{\alpha}^{*}=\min _{l \in L} C V a R_{\alpha}^{l}$, where the $\mathrm{CVaR}$ value along route $l$ in urban C\&DW transportation is defined using (3) and transformed as (4) [24].

$$
\begin{aligned}
& C V a R_{\alpha}^{l}=E\left[R^{l} \mid R^{l} \geq \operatorname{VaR}_{\alpha}^{l}\right], \\
& C V a R_{\alpha}^{l}=\lambda_{\alpha}^{l} V_{a}^{l} R_{\alpha}^{l}+\left(1-\lambda_{\alpha}^{l}\right) E\left(R^{l} \mid R^{l} \geq \operatorname{VaR}_{\alpha}^{l}\right),
\end{aligned}
$$




$$
\begin{aligned}
& \lambda_{\alpha}^{l}=\frac{F_{R^{l}}\left(\operatorname{VaR}_{\alpha}^{l}\right)-\alpha}{1-\alpha}=\frac{\operatorname{Pr}\left(R^{l} \leq C_{(r)}^{l}\right)}{1-\alpha}= \\
& =\frac{1}{1-\alpha}\left(\sum_{m=0}^{r} P_{(m)}^{l}-\alpha\right) \\
& E\left[R^{l} \mid R^{l} \geq \operatorname{VaR} R_{\alpha}^{l}\right]=\sum_{\eta} \eta f\left(\eta \mid R^{l} \geq V a R_{\alpha}^{l}\right)= \\
& =\sum_{\eta} \eta \frac{\operatorname{Pr}\left(R^{l}=\eta, R^{l} \geq \operatorname{VaR}_{\alpha}^{l}\right)}{\operatorname{Pr}\left(R^{l} \geq \operatorname{VaR}_{\alpha}^{l}\right)}= \\
& =\sum_{m=r+1}^{n_{l}} C_{(m)}^{l} \frac{P_{(m)}^{l}}{\sum_{m=r+1}^{n_{l}} P_{(m)}^{l}}
\end{aligned}
$$

Finally, we obtained the transformed CVaR value expression in (7) and proposed the CVaR value along path $l$ at the confidence level $\alpha$.

$$
C V a R_{\alpha}^{l}=\frac{1}{1-\alpha}\left[\left(\sum_{m=0}^{n_{l}} P_{(m)}^{l}-\alpha\right) \operatorname{VaR}_{\alpha}^{l}+\sum_{m=0}^{n_{l}} P_{(m)}^{l} C_{(m)}^{l}\right],
$$

$C \operatorname{VaR}_{\alpha}^{l}=$

$=\frac{1}{1-\alpha}\left[\left(\left(1-\sum_{m=r+1}^{n_{l}} P_{(m)}^{l}\right)-\alpha\right) C_{(r)}^{l}+\sum_{m=r+1}^{n_{l}} P_{(m)}^{l} C_{(m)}^{l}\right]=$.

$=C_{(r)}^{l}+\frac{1}{1-\alpha}\left[\sum_{m=r+1}^{n_{l}} P_{(m)}^{l}\left(C_{(m)}^{l}-C_{(r)}^{l}\right)\right]$

\section{OPTIMISATION PROGRAM}

\subsection{Model Transformation}

The optimisation problem in the urban C\&DW transport network involves determining a specific path $l$ indexed by nodes $i$ and $j$ that minimise $C V a R_{\alpha}^{l}$ of urban C\&DW transportation risk. In this subsection, a transforming method is described for the CRM to obtain an optimisation algorithm with which the minimum $C V a R_{\alpha}^{l}$ and its corresponding path $l$ can be achieved. Let $C_{(h)}$ denote the $h^{\text {th }}$ minimum accident consequence in the transport network $G(N, A), h \in\{0,1,2, \ldots, M\}, M=|A|$, $C_{(h)} \in\left\{C_{i j}:(i, j) \in A\right\}$. To determine the optimisation result for the proposed CRM $C V a R_{\alpha}^{*}=\min _{l \in L} C V a R_{\alpha}^{l}$, we define $\mathrm{P}_{(h)} \cdot X$ as follows:

$P_{(h)} \cdot X=\sum_{C_{i j}>C_{(h)},(i, j) \in A} P_{i j}\left(C i_{j}-C_{(h)}\right) x_{i j}$

The proposed CRM can be transformed as follows:

$$
\begin{aligned}
& C \operatorname{VaR}_{\alpha}^{*}=\min _{h} C \operatorname{VaR} R_{\alpha}^{h} \\
& \text { s.t. } \quad C V a R_{\alpha}^{h}=C_{(h)}+\frac{1}{1-\alpha} g^{h}, \\
& g^{h}=\min P_{(h)} \cdot X,
\end{aligned}
$$

$$
\begin{aligned}
& \Omega=\left\{X \mid \sum_{(i, j) \in A} x_{i j}-\sum_{(i, j) \in A} x_{j i}=\delta_{i}, \forall i, j \in N\right\}, \\
& \delta_{i}=\left\{\begin{array}{ll}
1 & i \in O \\
-1 & i \in D \\
0 & \text { otherwise }
\end{array},\right. \\
& x_{i j}=\left\{\begin{array}{l}
1, \text { if arc }(i, j) \text { is on the route } \\
0, \text { otherwise }
\end{array}\right. \text {, } \\
& \overline{\overline{P_{i j}}}=\left\{\begin{array}{ll}
P_{i j}\left(C_{i j}-C_{(h)}\right) & C_{i j}>C_{(h)}, \forall i, j \in N \\
0 & \text { otherwise }
\end{array} .\right.
\end{aligned}
$$

\subsection{Improved Algorithm}

The minimisation of $g^{h}$ can be regarded as finding the shortest path weighted by $P_{i j}\left(C_{i j}-C(h)\right)$. The value of $g^{h}$ can be calculated using the efficient Dijkstra algorithm. Consequently, we designed an algorithm by using three main steps, including detailed information depicted in Fig. 3.

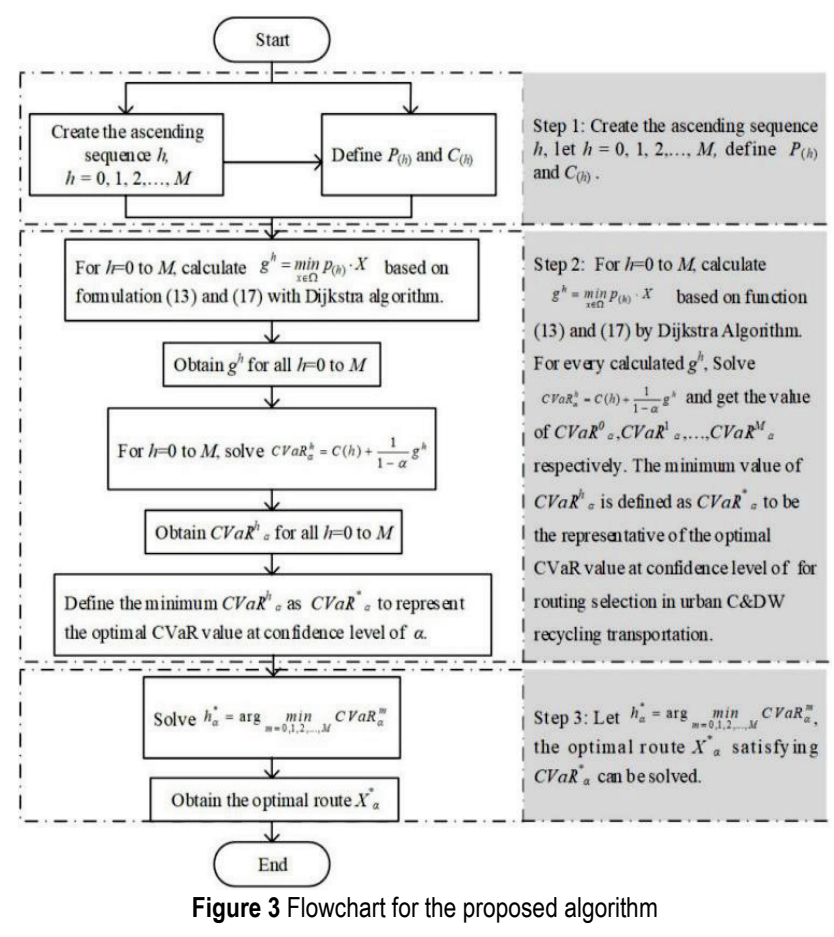

\section{CASE STUDY}

Big data technology plays a crucial role in the decision process. In this study, big data technology was used to determine the optimal routing decision for C\&DW transportation in Tongzhou District, Beijing, according to the CRM. Beijing has high traffic accident rates with severe consequences. According to reports, 3222 road traffic accidents resulted in 1376 deaths and a direct economic loss of 31455 million yuan in 2017 . The scenario in Tongzhou is considerably more serious than that in other areas of Beijing from the perspectives of both the accident rate and severity. In 2017, 456 road transport accidents occurred and the mortality rate per 10000 road vehicles was as high as 4.67 (the average mortality rate per 10000 road vehicles in Beijing city was 2.33). Numerous 
residential resettlement housing projects have been launched, which has led to increased threats to C\&DW transportation. Because of the densely distributed population and concentrated commuting trips in specific regions, the potential accident rate is high and the consequences are serious. Fig. 4 illustrates the location and some characteristics of the study area.

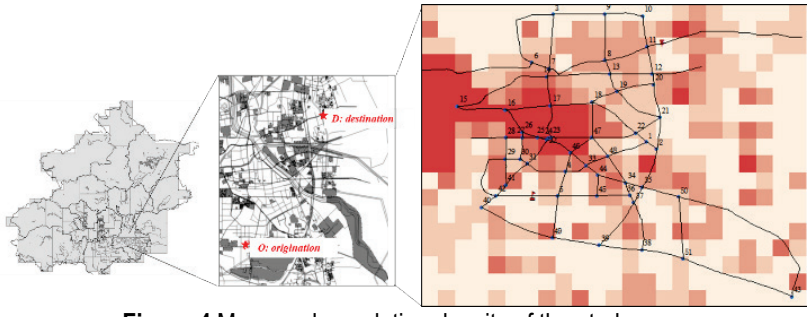

Figure 4 Maps and population density of the study area

\subsection{Preliminary Analysis with Big Data}

We considered the complexity of a dynamic transportation network and the historical-informationbased attributes of policy-making for applying the CRM to a static network.

\subsubsection{Transportation Network}

First, to obtain the transportation network of Tongzhou, OpenStreetMap was crawled using the quantum geographical information system and map data were obtained. The data included information on the road network as well as irrelevant information, such as the information on railways, river levees, underground passages, and footpaths. Then, ArcGIS was used to eliminate inappropriate elements and conduct a topology check on the crawled and modified road transportation network. The resulting C\&DW transport network had 53 nodes, 88 arcs, and a unique $(O, D)$ pair (Fig. 4). For a single C\&DW shipment, the objective was to determine the optimal path to move C\&DW from $O$ to $D$ such that the C\&DW recycling transportation risk measured using the CVaR was minimised.

\subsubsection{Accident Probability}

In practice, detailed data on C\&DW traffic accidents is unavailable. This data is replaced by the general road traffic accident probability because the probability of C\&DW transporting accidents is relatively high in sections with high road traffic accidents rates. Notably, $\varepsilon_{i j}$ is assumed as the number of road traffic accidents on arc $(i, j)$ in the following Poisson distribution with the expectation $\gamma_{i j}[23]$ :

$\gamma_{i j}=R_{v} \times L_{i j} \times V$

where $R_{v}$ is the road traffic accident rate, which represents the road traffic death rate per 10000 vehicles in Tongzhou District in 2017; $R_{v}=4.76 ; L_{i j}$ is the length of arc $(i, j)$, which is calculated according to the C\&DW transportation network; and $V$ is the average traffic volume, which is represented by the data for the suburbs in Beijing. A Poisson distribution is well known by its excellent applicability in describing the occurrence of rare random events in a certain period of time. Therefore, $\varepsilon_{i j}$ is assumed to follow Poisson distribution with the parameter $\gamma_{i j}$. The following formula can be used to calculate the road traffic rate on $\operatorname{arc}(i, j)$ :

$$
P_{i j}=1-\operatorname{Pr}\left\{\varepsilon_{i j}=0\right\}=1-\left\{\gamma_{i j} \frac{\varepsilon_{i j}}{\varepsilon_{i j} !}\right\} \times e^{-\gamma_{i j}}
$$

where $\varepsilon_{i j}=0$. Eq. (18) indicates that the road traffic accident rate of $\operatorname{arc}(i, j)$ is equal to 1 minus the probability of no accident occurring on the arc.

\subsubsection{Accident Consequences}

The other important parameter used in this paper is the accident consequence $C_{i j}$ caused by a C\&DW transportation accident. Considering the intention of safe transportation, the formula for accident consequences is designed according to the population density around arc $(i, j)$ and the traffic flow on arc $(i, j)$. The formula is expressed as follows:

$C_{i j}=w_{1}\left(\pi r^{2} \rho_{i j}\right)+w_{2} \frac{2 r}{L_{i j}} V_{i j}$

Notably, $\rho_{i j}$ is the population density of arc $(i, j), r$ represents the influencing radius of the C\&DW transport accident, and $w_{1}$ and $w_{2}$ denote different weights to population density and accident consequence affected by the traffic volume respectively. We obtained the data of population densities for the study area from the latest LandScan dataset, which is a community standard for global population distribution and is refreshed annually. In Fig. 5, the darker the colour, the higher is the population density. Unlike hazardous material road transportation, the influencing radius of $\mathrm{C} \& \mathrm{DW}$ traffic accidents is relatively limited. Compared with the population density, the traffic volume on arc $(i, j)$ has a higher effect on the severity of accident consequences. According to [23], $w_{1}$ and $w_{2}$ could affect the results of the model. We considered $r$ as $1 \mathrm{~km}$ and $w_{1}$ and $w_{2}$ as 1 and 8 , respectively.

\subsection{Numerical Results and Insights}

On the basis of the improved algorithm, the proposed CRM was implemented in Python 3.7 on a 3.1-GHz Intel Core i5 CPU computer system. Through simulation with Matlab R 2018a, various $\alpha$ values were divided into four intervals and the CRM was applied to the C\&DW transportation network of Tongzhou District. Finally, two different optimal routes were determined such that the relative value of $C V a R_{\alpha}$ was minimised. The optimal results and optimal routes for a minimised $\mathrm{CVaR}$ value and the shortest path are illustrated in Fig. 5. Detailed analyses and insights are given in the following text. 
Table 2 Optimal routes for different intervals of $\alpha$

\begin{tabular}{|c|c|c|c|c|c|}
\hline Optimal route & Intervals of $\alpha$ & $\operatorname{Arc} h_{\alpha}^{*}$ & Length $(\mathrm{km})$ & Given $\alpha$ & $C V a R_{\alpha}^{*}$ \\
\hline \multirow{3}{*}{ Path1 } & $(0,0.208]$ & $(0,42)$ & \multirow{3}{*}{18.171} & 0.2 & 1625.99 \\
\hline & $(0.208,0.276]$ & $(2,21)$ & & 0.25 & 1627.72 \\
\hline & $(0.276,0.991]$ & $(35,50)$ & & 0.9 & 1662.28 \\
\hline Path2 & $(0.991,1)$ & $(29,41)$ & 24.709 & 0.999 & 1707.30 \\
\hline Path3 & -- & -- & 13.171 & -- & -- \\
\hline
\end{tabular}

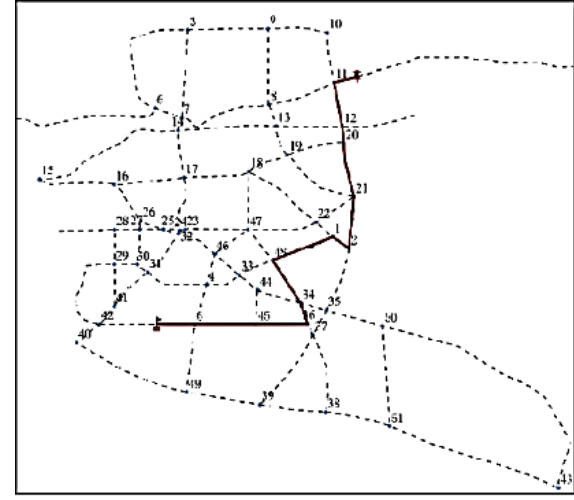

Path 1

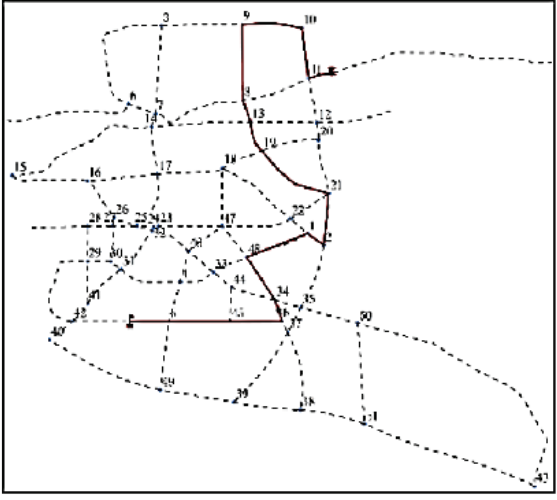

Path 2

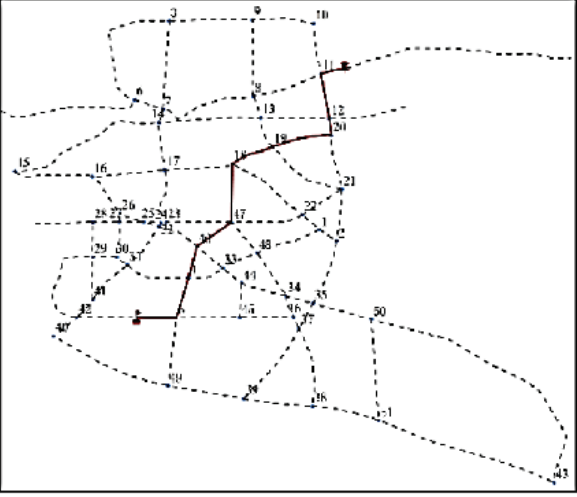

Path 3

Figure 5 Optimal routes that minimised the CVaR value and shortest path

\subsubsection{Optimal Routes under Different Risk-Averse Attitudes}

The values of the confidence coefficient $(\alpha)$ used were $(0,0.208),(0.208,0.276),(0.276,0.991)$, and $(0.991,1)$. People are proposed to be more risk-averse if $\alpha$ is closer to 1. The first optimal route of $0 \rightarrow 5 \rightarrow 45 \rightarrow 36 \rightarrow 34 \rightarrow 48 \rightarrow 1 \rightarrow 2 \rightarrow 21 \rightarrow 20 \rightarrow 12 \rightarrow 11 \rightarrow 52$ (Path1 in Fig. 6) is obtained when decision-makers' riskaverse attitudes are located in the first three intervals. For higher risk-averse attitudes, the other optimal route of $0 \rightarrow 5 \rightarrow 45 \rightarrow 36 \rightarrow 48 \rightarrow 1 \rightarrow 2 \rightarrow 21 \rightarrow 19 \rightarrow 13 \rightarrow 8 \rightarrow 9 \rightarrow 10 \rightarrow$ $11 \rightarrow 52$ (Path2 in Fig. 6) is decided. From the numerical values of $\alpha$ inside the four intervals, we obtained the corresponding values of $C V a R_{\alpha}^{*}$. The shortest path $0 \rightarrow 5 \rightarrow 4 \rightarrow 46 \rightarrow 47 \rightarrow 18 \rightarrow 19 \rightarrow 20 \rightarrow 12 \rightarrow 11 \rightarrow 52 \quad$ was obtained (Path3 in Fig. 6) when only the distance was considered with the traditional Dijkstra algorithm.

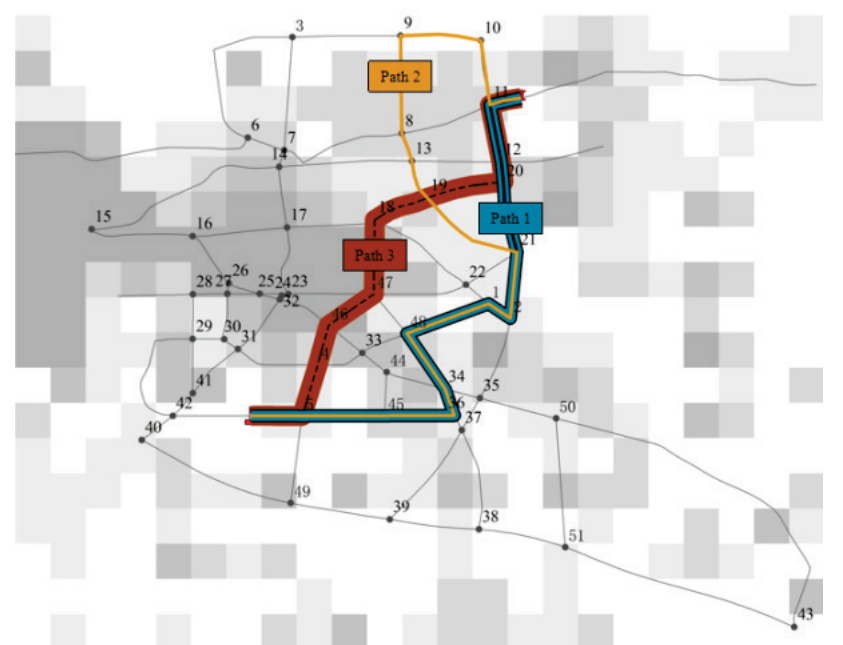

Figure 6 Characteristics of urban C\&DW optimal transportation routes

Fig. 6 illustrates different characteristics of the three optimal paths. First, the optimal routes calculated with the proposed CRM (Path 1 and Path 2) are considerably longer than the shortest path (Path 3). Moreover, Path 2 has more detours than Path 1 does. With regard to the population density, the two paths, especially Path 2, are along areas with low population density. However, Path 3 directly passes through the most populated area. Thus, decisionmakers' risk-averse attitudes have a critical influence on the configurations of optimal C\&DW transportation routes. In other words, people with rigorous risk-averse attitudes and abundant knowledge of transportation safety prefer to sacrifice transportation efficiency and select less densely populated roads than a straight route. Second, the lengths of Path 1 and Path 2 are considerably larger than the length of Path 3. Moreover, Path 2 is the longest route for C\&DW transportation between the defined $(O, D)$ pair. Therefore, the higher risk-averse the decision-makers are, the higher is the possibility that they would select long routes even if this means additional energy consumption, exhausted emission, and transportation cost. Therefore, decisionmakers should trade-off between risk prevention and economic and environmental benefits during C\&DW transportation. The higher the degree of risk aversion degree, the more inclined are the decision-makers to select a long path to ensure the safety of human life.

Awareness should be created on risk control to improve decision-makers' risk avoidance. The selection of an optimal path for C\&DW transportation would prevent transportation accidents in high-risk areas, reduce the potential severe consequences of traffic accidents, and guarantee the personal safety of urban residents.

\subsubsection{Sensitive Analysis for Different Risk Aversion Attitudes of Decision-Makers}

We determined which confidential interval results in the highest sensitivity to decision-makers. We explored what type of measures should be taken to enhance decision-makers' degree of risk aversion for ensuring that urban C\&DW is transported along a safe path. Moreover, further research was conducted on sensitivity to analyse the relationship between decision-makers' risk-averse attitude $(\alpha)$ and the minimum value of $C V a R_{\alpha}^{*}$. 
As presented in Tab. 2 and Fig. 7, the minimum value of $C V a R_{\alpha}^{*}$ increases with increasing $\alpha$. However, the rate of increase of $C V a R_{\alpha}^{*}$ is very slow if $\alpha$ is located in low confidential intervals, where no change occurs in the optimal route selection. By contrast, a sharp upward trend and a considerable change in the rate of $\mathrm{CVaR}_{\alpha}^{*}$ is observed if $\alpha$ is located in the high intervals $(0.991-1)$, where significant change occurs (last subgraph of Fig. 7). Thus, the higher the degree of decision-makers' risk aversion, the higher is the sensitivity of the optimal route selection. Therefore, maintaining a high degree of risk aversion is conducive to maintaining high consciousness, initiative, and durability in safe urban C\&DW routing decisions. This conclusion further verifies and extends the existing study [25] to the field of C\&DW transportation.

According to the aforementioned analysis, for typical low-probability-high-consequence events, such as urban C\&DW traffic accidents, a relatively high risk-averse degree must be set to help decision-makers actively minimise the probabilities and consequences of potential risks through optimal path selection.

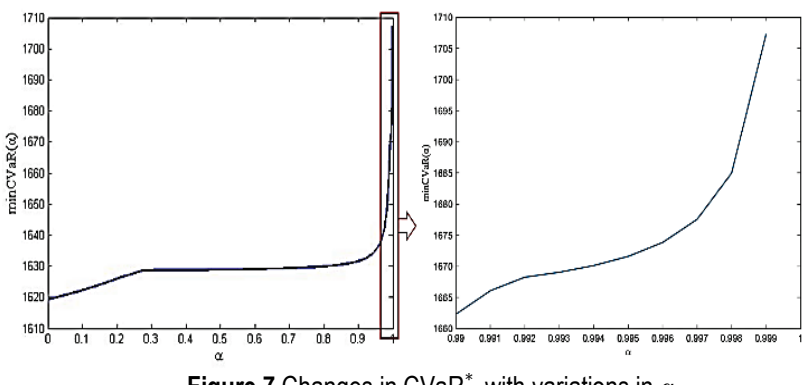

Figure 7 Changes in $\mathrm{CVaR}_{\alpha}^{*}$ with variations in $\alpha$

\section{CONCLUSIONS AND FUTURE STUDIES}

To address the challenges of urbanisation, promotion and maintenance of safe cities and communities has been set up as one of the sustainable development goals by the United Nations. However, limited attention on and understanding of the potential accident risks and consequences are fundamental reasons for urban C\&DW transportation becoming an obstacle to safety. To improve transportation safety, control the transportation risk, and accumulate theoretical support for policy-making, we used routing design. We attempted to develop an optimal routing model for $\mathrm{C} \& \mathrm{DW}$ transportation based on the CVaR criterion by evaluating decision-makers' various risk-averse attitudes. With big data technology, companies and governments can guide the behaviours of decisionmakers. Therefore, we propose a multistructure dataset and a cross-research framework that combines the traditional optimisation model with big data technology to extend theoretical studies in the field of safe routing decisions for C\&DW.

To solve the proposed CRM, an improved algorithm was introduced and conclusions were drawn according to the big data analysis for Tongzhou District, Beijing. First, the optimal route decision for urban C\&DW recycling transportation was considerably affected by the degree of risk aversion of decision-makers, which indicated that the degree of risk aversion is crucial for making C\&DW transportation routing decisions from the perspective of people-oriented risk control. The higher the degree of risk aversion, the higher is the preference of decision-makers to reduce accident incidences and consequences and improve road transportation safety. However, the optimal route in such cases would be more circuitous and longer. Second, the higher the degree of risk aversion of decision-makers, the higher is the sensitivity in optimal path selection. Small changes in the degree of risk aversion result in large changes in the optimal path decision. According to these conclusions, we recommend suggestions for managers from administrations and enterprises. Governments should develop policies and regulations to maximise the level of urban C\&DW transportation safety and minimise the probability and consequences of potential traffic accidents by improving the supervision strength, enhancing the technology monitoring and enforcement, and executing traffic control policies on some hazardous roads with high accident rates. Managers of C\&DW transportation companies can use technical support techniques, such as global positioning systems, automobile sensors, and vehicle navigation, to assist their drivers in selecting optimal routes. In addition, managers are responsible for the risk-related training programme to educate their employees. The training programme should enhance their risk aversion degree, consciousness, and initiative for avoiding C\&DW transportation risks.

For reducing the complexity of the proposed model, time-varying conditions and multiple vehicle trips were not considered. These aspects can be considered in future studies. Big data analytics can be used to achieve meaningful research for theoretical expansion and practice guidance.

\section{Acknowledgements}

This research has been sponsored by Beijing Social Science Foundation (grant number 17JDGLB015, 16YJC049, 18GLB020), Social Scientific Project of Beijing Education Commission (grant number SM201910017002) and Beijing Great Wall Scholar Training Program (CIT\&TCD20180314).

\section{REFERENCES}

[1] Jia, S. W. \& Yan, G. (2018). Dynamic simulation of construction waste management model under combination policies. Systems Engineering - Theory \& Practice, 38(11), 2966-2978.

https://doi.org/10.12011/1000-6788(2018)11-2966-13.

[2] Rahimi, M. \& Ghezavati, V. (2018). Sustainable multiperiod reverse logistics network design and planning under uncertainty utilizing conditional value at risk $(\mathrm{CVaR})$ for recycling construction and demolition waste. Journal of Cleaner Production, 172, 1567-1581. https://doi.org/10.1016/j.jclepro.2017.10.240.

[3] Illenberger, J., Flotterod, G., \& Nagel, K. (2011). A model of risk-sensitive route-choice behavior and the potential benefit of route guidance. IEEE Transactions on Intelligent Transportation Systems, 12(2), 384-389. https://doi.org/10.1109/TITS.2011.2105266.

[4] Deery, H. A. \& Fildes, B. N. (1999). Young novice driver subtypes: relationship to high-risk behavior, traffic accident record, and simulator driving performance. Human Factors, 41(4), 628-643. https://doi.org/10.1518/001872099779656671

[5] Stanojević, D., Stanojević, P., Jovanović, D., et al. (2018). Impact of riders' lifestyle on their risky behavior and road traffic accident risk. Journal of Transportation Safety \& Security. https://doi.org/ 10.1080/19439962.2018.1490367 
[6] Hao, W. Y., Wang, S., Shi, W. Z., et al. (2017). Optimizing GPS-guidance transit route for cable crane collision avoidance using artificial immune algorithm. GPS Solutions, 21(2), 823-834. https://doi.org/10.1007/s10291-016-0573-6

[7] Toth, P. \& Vigo, D. (2014). Vehicle Routing: Problems, Methods, and Applications, ( ${ }^{\text {nd }}$ Ed). Philadelphia, PA: Society for Industrial and Applied Mathematics.

[8] Zhao, P. X., Luo, W. H., \& Han, X. (2019). Time-dependent and bi-objective vehicle routing problem with time windows. Advances in Production Engineering \& Management, 14(2), 201-212. https://doi.org/10.14743/apem2019.2.322

[9] Zhang, X. N. \& Fan, H. M. (2018). Optimization for multitrip vehicle routing problem with fuzzy demands considering time window preference. Computer Integrated Manufacturing Systems, 24(10), 2461-2477. https://doi.org/10.13196/j.cims.2018.10.009

[10] Zhao, P. X., Gao, W. Q., Han, X., et al. (2019). Bi-objective collaborative scheduling optimization of airport ferry vehicle and tractor. International Journal of Simulation Modelling. 18(2), 355-365. https://doi.org/10.2507/IJSIMM18(2)CO9

[11] Inmaculada, R. M., Juan-JosêS, G., \& Hande, Y. (2019). The periodic vehicle routing problem with driver consistency. European Journal of Operational Research, 273(2), 575-584. https://doi.org/ 10.1016/j.ejor.2018.08.032

[12] Liu, Y., Li, Y., \& Hu, L. (2018).Departure time and route choices in bottleneck equilibrium under risk and ambiguity. Transportation Research Part B: Methodological, 117, 774793. https://doi.org/10.1016/j.trb.2017.09.007

[13] Zhao, J. H. \& Ke, G. Y. Incorporating inventory risks in location-routing models for explosive waste management. International Journal of Production Economics, 193, 123136. https://doi.org/10.1016/j.ijpe.2017.07.001

[14] Wang, N., Zhang, M., Che, A., et al. (2018). Bi-objective vehicle routing for hazardous materials transportation with no vehicles travelling in echelon. IEEE Transactions on Intelligent Transportation Systems, 19(6), 1867-1879. https://doi.org/10.1109/TITS.2017.2742600.

[15] Payyanadan, R. P., Maus, A., Sanchez, F. A., et al. (2017). Using trip diaries to mitigate route risk and risky driving behavior among older drivers. Accident Analysis and Prevention, 106, 480-491. https://doi.org/10.1016/j.aap.2016.09.023

[16] Gong, D., Tang, M., Liu, S., et al. (2019). Achieving sustainable transport through resource scheduling: a case study for electric vehicle charging stations. Advances in Production Engineering \& Management, 14(1), 65-79. https://doi.org/10.14743/apem2019.1.312

[17] Tang, M., Gong, D., Liu, S., et al. (2017). Finding Key Factors for electric vehicle charging station location: a simulation and ANOVA approach. International Journal of Simulation Modelling, 16(3), 541-554. https://doi.org/ 10.2507/IJSIMM16(3)C015

[18] Du, J. B., Li, Q., Qiao, F. X., et al. (2018). Estimation of vehicle emission on mainline freeway under isolated and integrated ramp metering strategies. Environmental Engineering and Management Journal, 17(5), 1237-1248. https://doi.org/10.30638/eemj.2018.123

[19] Chen, J. M. (2018). On exactitude in financial regulation: value-at-risk, expected shortfall, and expectiles. Risks, 6(2), 61-88. https://doi.org/10.3390/risks6020061

[20] Kang Y., Batta, R., \& Kwon, C. (2014) Value-at-risk model for hazardous material transportation. Annals of Operations Research, 222(1), 361-387. https://doi.org/10.1007/s10479-012-1285-0

[21] Hosseini, S. D. \& Verma, M. (2017). A value-at-risk (VaR) approach to routing rail hazmat shipments. Transportation Research Part D: Transport and Environment, 54, 191-211. https://doi.org/10.1016/j.trd.2017.05.007

[22] Nocera, J. (2009, January 4). Risk Mismanagement. http://ww.nytime.com/2009/01/04/magazine/04risk-t.html.
[23] Toumazis, I. \& Kwon, C. (2013). Routing hazardous materials on time dependent networks using conditional value-at-risk. Transportation Research Part C: Emerging Technologies, 37, 73-92. https://doi.org/10.1016/j.trc.2013.09.006

[24] Hosseini, S. D. \& Verma, M. (2018). Conditional value-atrisk (CVaR) methodology to optimal train configuration and routing of rail hazmat shipments. Transportation Research Part B: Methodological, 110, 79-103. https://doi.org/10.1016/j.trb.2018.02.004

[25] Cao, H., Fan, T. J., \& Liu, L. P. (2017). Routing the road and railway intermodal transportation of hazmat based on CVaR. Operations Research and Management Science, 26(6), 4148. Retrieved from http://www.jorms.net/CN/10.12005/ orms.2017.0135.

[26] Faghih-Roohi, S., Ong, Y. S., Asian, S., et al. (2016). Dynamic conditional value-at-risk model for routing and scheduling of hazardous material transportation networks. Annals of Operations Research, 247(2), 715-734. https://doi.org/10.1007/s10479-015-1909-2

[27] Alex, N., Indratmo, Ben, D., et al. (2019). Systematic review of the literature on big data in the transportation domain: concepts and applications. Big Data Research. https://doi.org/10.1016/j.bdr.2019.03.001

[28] Lin, Y., Wang, P., \& Ma, M. (2017). Intelligent transportation system (ITS): concept, challenge and opportunity. IEEE International Conference on Big Data Security on Cloud. IEEE. https://doi.org/10.1109/BigDataSecurity.2017.50

[29] Dobre, C. \& Xhafa, F. (2014). Intelligent services for Big Data science. Future Generation Computer Systems, 37(2), 267-281. https://doi.org/10.1016/j.future.2013.07.014

[30] Ta-Shma P., Akbar A., \& Gerson-Golan G., et al. (2018). An ingestion and analytics architecture for IoT applied to smart city use cases. IEEE Internet of Things Journal, 5(2), 765S774. https://doi.org/ 10.1109/JIOT.2017.2722378

[31] Lakshmanaprabu, S. K., Shankar, K., Sheeba, S., et al., (2019). An effect of big data technology with ant colony optimisation based routing in vehicular ad hoc networks: towards smart cities. Journal of Cleaner Production, 217. 584-593. https://doi.org/10.1016/j.jclepro.2019.01.115

[32] Stanley, K., Yoo, E. H., Paul, T., et al. (2018). How many days are enough? Capturing routine human mobility. International Journal of Geographical Information Science, 32(7), 1485-1504 https://doi.org/10.1080/13658816.2018.1434888

[33] Rockafellar, R.T. \& Uryasev, S., (2000). Optimization of conditional value-at-risk. Journal of Risk, 2(3), 21-42. https://doi.org/10.21314/JOR.2000.038

\section{Contact information:}

Ying QIU, PhD, Lecture

Beijing Institute of Petrochemical Technology,

School of Economics and Management,

No.19 Qingyuan North Road, Daxing District, 102617, Beijing, China

E-mail: qiuying@bipt.edu.cn

Xinna ZHAO, PhD, Lecture

(Corresponding author)

Beijing Institute of Petrochemical Technology,

School of Economics and Management,

No.19 Qingyuan North Road, Daxing District, 102617, Beijing, China

E-mail: zhaoxinna@bipt.edu.cn

Xiaohong ZHANG, PhD, Professor

Beijing Institute of Petrochemical Technology,

School of Economics and Management,

No.19 Qingyuan North Road, Daxing District, 102617, Beijing, China

E-mail: zhangxiaohong@bipt.edu.cn 